\title{
A SURVEY OF DESIGN REVIEWS: UNDERSTANDING DIFFERENCES BY DESIGNER-ROLES AND PHASE OF DEVELOPMENT
}

\author{
Chen, James; Zucco, Gustavo; Olechowski, Alison \\ Department of Mechanical \& Industrial Engineering, University of Toronto
}

\begin{abstract}
In this paper, we present the results of a survey of new product development practitioners regarding their design review experiences. We surveyed 128 product development professionals on their experience and preferences in design reviews. We found that the goals and type (location / synchronicity) of design reviews change over the course of a product development project. We found that the majority of design review meetings continue to be held as co-located, live, in-person meetings. For reviewing 3D models, we found that a native CAD package (rather than a viewer, or fixed views, or a physical prototype) is the most commonly used tool. We found a difference between Designers (more likely to be product engineers) and Non-Designers and their access to CAD software, as well as their preference for which tool to use at the design review for 3D model evaluation. We hope that our findings spark future work related to better understanding design reviews and design reviewers in context. Design reviews are an important part of industrial product development processes, so we believe future studies have a large potential to improve these design activities
\end{abstract}

Keywords: Decision making, Information management, New product development, Design review

\section{Contact:}

Olechowski, Alison

University of Toronto

Mechanical \& Industrial Engineering

Canada

olechowski@mie.utoronto.ca

Cite this article: Chen, J., Zucco, G., Olechowski, A. (2019) 'A Survey of Design Reviews: Understanding Differences by Designer-Roles and Phase of Development', in Proceedings of the 22nd International Conference on Engineering Design (ICED19), Delft, The Netherlands, 5-8 August 2019. DOI:10.1017/dsi.2019.281 


\section{INTRODUCTION}

The practice of design review (DR) is a ubiquitous part of the modern product development process in industry (Adams et al., 2016). DR activities involve understanding and assessing the design of a product or process against a set of requirements, defining improvements or subsequent actions, and confirming readiness for the next stage. The set of requirements may be formal (e.g. documents and specifications) or informal (tacit, from experience or judgment) and are derived from product targets and lessons learned (previous experience and knowledge). The assessment is performed by first gaining an understanding of the design elements with the aid of their representation in the form of models (of interest to this study - 3D models), drawings, or list of materials. Next, the reviewers judge the fit for purpose of the product with respect to the set of requirements. Often, the many decisions (Krishnan and Ulrich 2001) that must be made about the product are discussed and determined at the DR.

Despite the pervasiveness of DR as major points of influence in a new product development process, little empirical understanding of DR has been achieved (Barczak 2012, Schmidt et al., 2009).

The focus of this paper is on Design Reviews where computer-aided design (CAD) models are the design representation under review. This research aims to reveal new information about how the phase of the review and the role of the reviewer relate to other review characteristics. In effect we seek to show that design reviews involving CAD models differ in ways that may be important for engineering designers and managers.

\section{LITERATURE}

Motivated by the same goal of better understanding new product development design reviews, Schmidt et al., (2009) surveyed 425 product developers. The authors focused on three design reviews in the new product development process: initial screen, prior to development and testing, and prior to commercialization. This study focuses on criteria used for evaluation during the design review, and finds difference in number and type of criteria between radical and incrementally innovative projects.

Germani et al., (2012) defined four types of collaboration in design: synchronous and co-located (e.g. face-to-face meetings); synchronous and remote (e.g. videoconference and web-sharing); asynchronous and co-located (e.g. routine design activity of a team in the same company); and asynchronous and remote (e.g. design activity of a team involving multiple companies or locations).

The type of design review meeting (co-located, remote, synchronous) influences the type of communication modes, and the number of reviewers available at the review. Investigating the role of communication at formal design reviews, Ostergaard et al., (2005) conducted a controlled user study of the effectiveness of various types of communication. The goal of the exercise was for subjects to identify design flaws. The authors found that groups are twice as effective as individuals at design reviews, providing evidence that current industry practice of teamwork in new product development is appropriate. The study further finds that in-person, face-to-face communication was perceived as more effective than speech-only communication, and speech-only communication was perceived as more effective than text-only communication. Although perceived as more effective, the authors did not find a significant difference in the measured effectiveness of design reviews under these different communication modes.

Again, the type of review is important given the findings of studies investigating individual and team reviewers, and access to information. Wetmore III et al., (2010) studied groups' ability to identify design problems, and the influence of group familiarity and information access. They found that the less information is shared among team members, the less likely the team will be able to locate the design problem based on the available information. The study did not find group familiarity to have a significant effect on the design review activity, which may have interesting implications for the increasingly diverse and global make-up of design review teams.

Design Reviews play an important role in generating and managing knowledge about a product within a company or its partners (suppliers and customers involved on the product design), for they "provide a unique information synchronization point in the development" where the progress can be collaboratively evaluated (Huet et al., 2007). This paper evaluates extensively the task of minute taking as means of generating and managing knowledge. Annotations are cited as an important part of the engineering activity and that engineers respect and value the actions list from the DR sessions (in form of annotation and minutes). 
In one of the few references about the impact of shared 3D visual representation, citing previous research on collaborative virtual environments, Gul et al., (2013) stated that the participant's ability to manipulate shared 3D models in real-time from their own viewpoint facilitates the coordination of collaborative design, rapid consensus, and decision making. The present study aims to address a gap in the literature: an improved characterization of design reviews which involve the review of 3D models.

\section{METHOD}

We created a survey aimed at professionals from companies of various industrial sectors that perform product design. The subject of design may be mechanical of any type (machine, die, component, subassembly, complete complex product), however the product must be representable for visualization in $2 \mathrm{D}$ and $3 \mathrm{D}$. The survey is targeted at professionals that participate actively in design review sessions at their companies. The respondents were asked to respond to the questions based on their most recent design review experience.

A pre-trial of the survey was conducted with eight respondents to identify any problems with the survey instrument before it was released to a wider audience. The pre-trial consisted of a preliminary version of the survey questions, presented to a selected group of designers, design researchers and industry professionals. Feedback on the language and understanding of the survey questions was obtained through e-mails and interviews, guiding minor changes. The data from the pre-trial was not included in the survey results, nor were the pre-trial participants invited to answer the final survey.

\subsection{Distribution}

The survey was distributed to industry contacts through email, social networks, and invitations in CAD discussion boards and newsletters. From the total of 128 responses received, the vast majority (107 responses, $78.1 \%$ ) originated from an anonymous link in an invitation for participation distributed via email, which encouraged snowball sampling. Posts and direct messages in social networks such as Facebook, Reddit and LinkedIn were also used for inviting participants. From the social networks, LinkedIn generated the most responses (21, $15.3 \%$ of the total). The remaining responses originated from a post on the GrabCAD Forum, a CAD users' forum and model sharing platform, and from an invitation sent out in GrabCAD's weekly newsletter to registered members.

\subsection{Design}

The style of the questions was conversational and colloquial, so as to establish a personal connection to the respondents. After an initial consent page explaining the survey, the time required, and the privacy and security of the data, the questionnaire was presented in three parts: (1) The background of the respondents, their company and how they participate in design review sessions; (2) The practices used during design review sessions in which they participate; (3) Common issues that may happen at design review sessions and can be influenced by the visual representation and collaboration tools used by the team. The survey was available in both English and Portuguese (the respondent's choice).

\subsection{Analysis}

The survey responses were exported from the Qualtrics platform as a .csv file, then loaded into R for analysis. Analysis began with summarizing key demographics, including respondent industry sectors, location, core functional roles, CAD access, stages of Product Development (PD), and DR meeting type. CAD Designer/Others status were differentiated by the respondents' answer to the survey question shown in Figure 1. CAD Designers self-identified as such, while those labeled as "Others" included respondents with no CAD experience at all, or having a basic understanding of CAD and use CAD viewers but do not design in a professional capacity.

Shown in Figure 2, we combined responses to create a 3-item variable for design process phase based on the phases of Ulrich and Eppinger (2015); Conceptual Design consists of "planning" and "concept development"; Detail Design consists of "system-level design" and "detail design"; Production consists of "testing and refinement" and "product ramp-up." 
What is your experience in using CAD for viewing or editing $3 \mathrm{D}$ models?

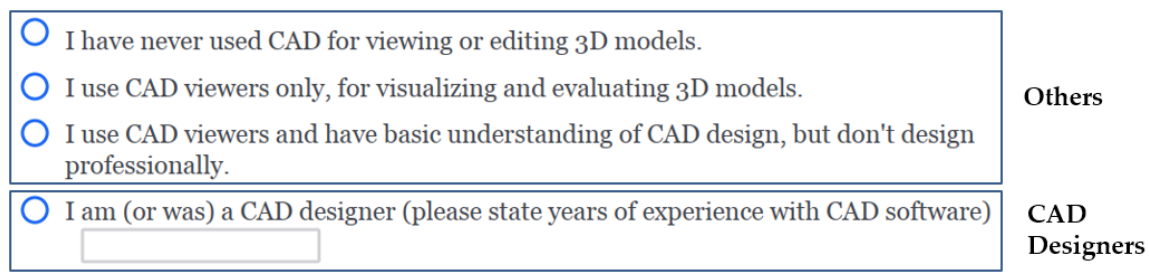

Figure 1. Survey question used to differentiate designerlothers

At which stage of the product development process (PDP) did the review take place?

\begin{tabular}{|ll|}
\hline & Planning / Product definition (early requirements) \\
$\bigcirc$ & Concept Development \\
\hline \hline O & System-Level Design \\
$\bigcirc$ & Detailed Design \\
$\bigcirc$ & Testing and Refinement \\
\hline \hline & Production Ramp-Up and Serial Production \\
\hline
\end{tabular}

Conceptual Design

Detail Design

Production

Figure 2. Survey question used to create the design process phase variable

\section{RESULTS}

\subsection{Overview of dataset}

Tables 1 and 2 provide summary statistics for context variables and designer review variables. Results show that the majority of responses are from Brazil and the automotive sector, at $60.6 \%$ and $77.3 \%$ respectively. This is a result of the respondent recruitment effort and the snowball sampling strategy. Implications of this bias are discussed below in the limitations section. The majority of respondents, $88.1 \%$, responded to the survey with an early design review stage experience in mind, either conceptual or detail design. Co-located, in-person meetings were the most common meeting types identified by the respondents.

Table 1. Summary statistics of full survey response set.

\begin{tabular}{lll} 
Variable & Number of Observations & Percentage \\
\hline Overall Total & 128 & \\
\hline Countries & 127 & \\
South America* & 77 & $60.6 \%$ \\
North America & 34 & $26.8 \%$ \\
Europe & 12 & $9.4 \%$ \\
Asia & 4 & $3.1 \%$ \\
\hline Industry & 128 & \\
Automotive & 99 & $77.3 \%$ \\
Medical & 11 & $8.6 \%$ \\
Aerospace/Defense & 5 & $3.9 \%$ \\
Other & 13 & $10.1 \%$ \\
\hline Functional Area (core role) & 127 & \\
Product Engineering & 42 & $33.1 \%$ \\
Project Management & 29 & $22.8 \%$ \\
Quality Assurance & 24 & $18.9 \%$ \\
Manufacturing Engineering & 14 & $11 \%$ \\
Purchasing/Sales & 8 & $6.3 \%$ \\
Product Design & 6 & $4.7 \%$ \\
Production/Logistics & 4 & $3.1 \%$ \\
\hline
\end{tabular}


Table 2. Summary statistics of full survey response set for design review variables of interest.

\begin{tabular}{lll} 
Variable & Number of Observations & Percentage \\
\hline Phase of PD & 126 & \\
Conceptual Design & 43 & $34.1 \%$ \\
Detail Design & 68 & $54.0 \%$ \\
Production & 15 & $11.9 \%$ \\
\hline CAD Designer Status & 128 & \\
CAD Designer & 44 & $34.4 \%$ \\
Others & 84 & $65.6 \%$ \\
\hline CAD Access & 96 & \\
Had access & 57 & $59.4 \%$ \\
No access & 39 & $40.6 \%$ \\
\hline Meeting Type & 128 & $52.3 \%$ \\
Co-located live meeting & 67 & $33.6 \%$ \\
Mixed live and remote & 43 & $9.4 \%$ \\
Remote meeting (online) & 12 & $4.7 \%$ \\
Asynchronous (no meeting held) & 6 & \\
\hline
\end{tabular}

\subsection{Characterizing design reviews by phase}

Figure 3 characterizes design reviews by phase with respect to design review location, goal, and main tool used during the meeting.

Respondents were allowed to select multiple options regarding the question of design review goals. The options were "brainstorming concept ideas," "communicating a design change," "consolidating design from different areas," "making decision on maturity level," and "making decision on investments." If a participant selected multiple options, each of their selections were averaged. The figure shown is therefore generated using the weight-adjusted scores for each goal.

We can see that as the project matures, a larger proportion of the meetings move from live co-located meetings to mixed, online remote or asynchronous meetings. We postulate this is due to the fact that as a project matures and more parties become involved, live co-located meetings become more difficult to conduct and logistically challenging. Another interesting observation is that the mixed live/remote meeting type stays relatively consistent throughout the three design phases. This may be because many companies are habituated to online meetings as a medium for conducting design reviews.

Looking at the second row in Figure 3, the goal of the design reviews, we see brainstorming trend downwards as the product development progresses to later stages. In fact, we tested the dichotomous variable of "brainstorming" as goal of phase across the three phases, and found a significant chi-squared test of independence result $\chi^{2}(4, \mathrm{~N}=182)=10.68, \mathrm{p}<.05$. This result gives us confidence in our data set and interpretation by our survey respondents; brainstorming is a key part of ideation, which occurs during conceptual design. We also see that the most common goal of a design review during the detailed design phase is to perform a gate review, while for the production phase it is to communicate a design change.

Next, we look at the main tool for 3D visualization used during design review meetings in the three phases. Native 3D CAD remains the most commonly used tool during design reviews. Physical prototype is much more popular in the production phase compared to the two design phases.

\subsection{Understanding design review participants: CAD designers and others}

Figure 4 shows the breakdown of CAD access, core roles, and main visual representation tool used during DR between CAD Designers and Others.

We first test for significant differences in CAD access between CAD Designers and Others and find that CAD Designers exhibit a higher likelihood of having access to CAD systems in their companies. A chi-squared test of independence was significant, $\chi^{2}(1, \mathrm{~N}=95)=5.20, \mathrm{p}<.05$, suggesting there is a significant difference in CAD Access between CAD Designers and Others.

Next, we constructed a dichotomous variable of core role, separating the role of "product engineering" from the rest. We found that, using a chi-squared test of independence, $\chi^{2}(1, \mathrm{~N}=127)=8.72, \mathrm{p}<.01$, 


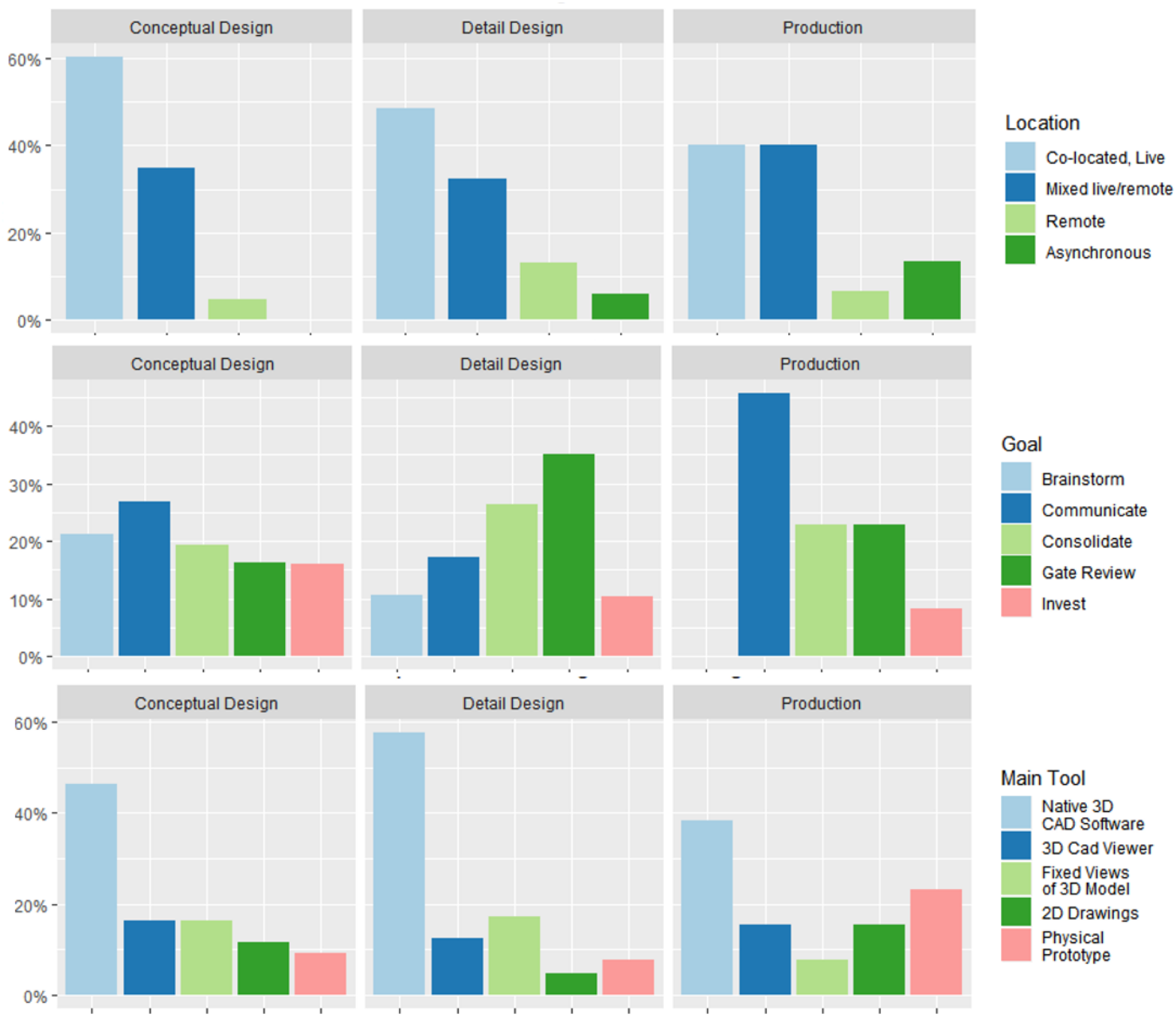

Figure 3. Survey responses tabulated by the phase of product design process in which the design review occurred.

CAD Designers are significantly more likely to be involved in product engineering roles compared to Others. From the data, we can also see that Others tend to more likely be involved in roles such as quality assurance, purchasing/sales, project management, and operations.

Lastly, on the bottom row of Figure 4, we see differences in the preferences for visual representation between CAD Designers and Others. CAD Designers rely on 3D CAD during design reviews more so than Others, either using native CAD software or $3 \mathrm{D}$ CAD viewers. It is also interesting to note that many more Others reported using physical prototypes as their main tool for visual representation compared to CAD Designers, at $13.8 \%$ vs $4.2 \%$ respectively.

\section{DIscussion}

Design reviews are an integral part of the engineering design process. This study demonstrated that there are some key differences separating CAD Designers' and Others' experiences in design reviews. CAD Designers and Others - all participants in design review exercises - exhibit different preferences for visual representation tools, and tend to occupy different roles in organizations.

The skewed distribution of roles between CAD Designers and Others sparks new questions about the composition of design review teams. Previous studies have pointed to the benefit of cross-functionality in new product development teams (Edmondson and Nembhard 2009), including knowledge sharing, learning, and the consideration of wider range of perspectives. The diversity of expertise could benefit teams in terms of variety of perspective, but might also prevent effective communication or cause tension between sub-teams as they have different mental models. Digital and physical models of the design may be critically important in this scenario given their potential as boundary-spanning objects (Carlile 2002). For this reason, our understanding of the 3D CAD model access and preferences may reveal important differences to consider. 

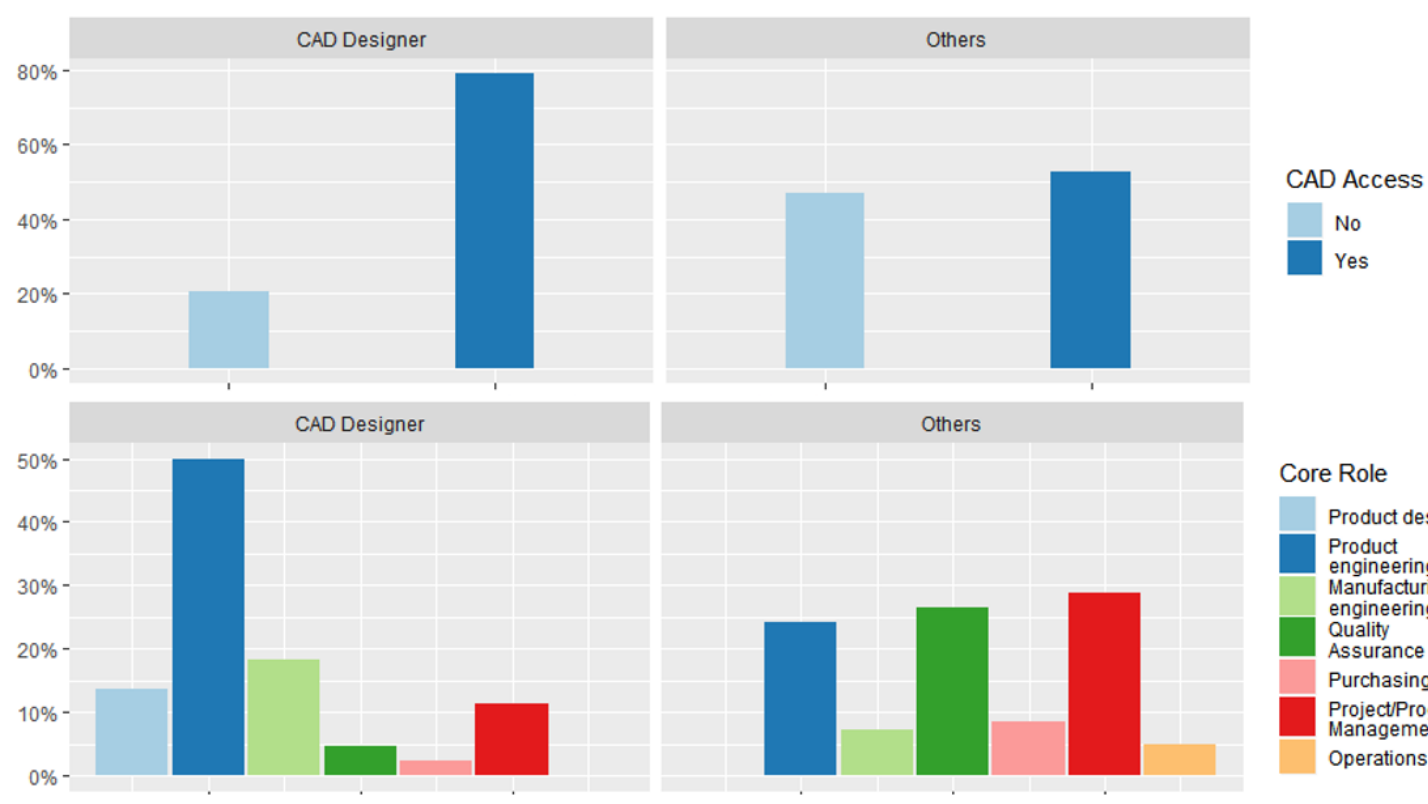

Core Role
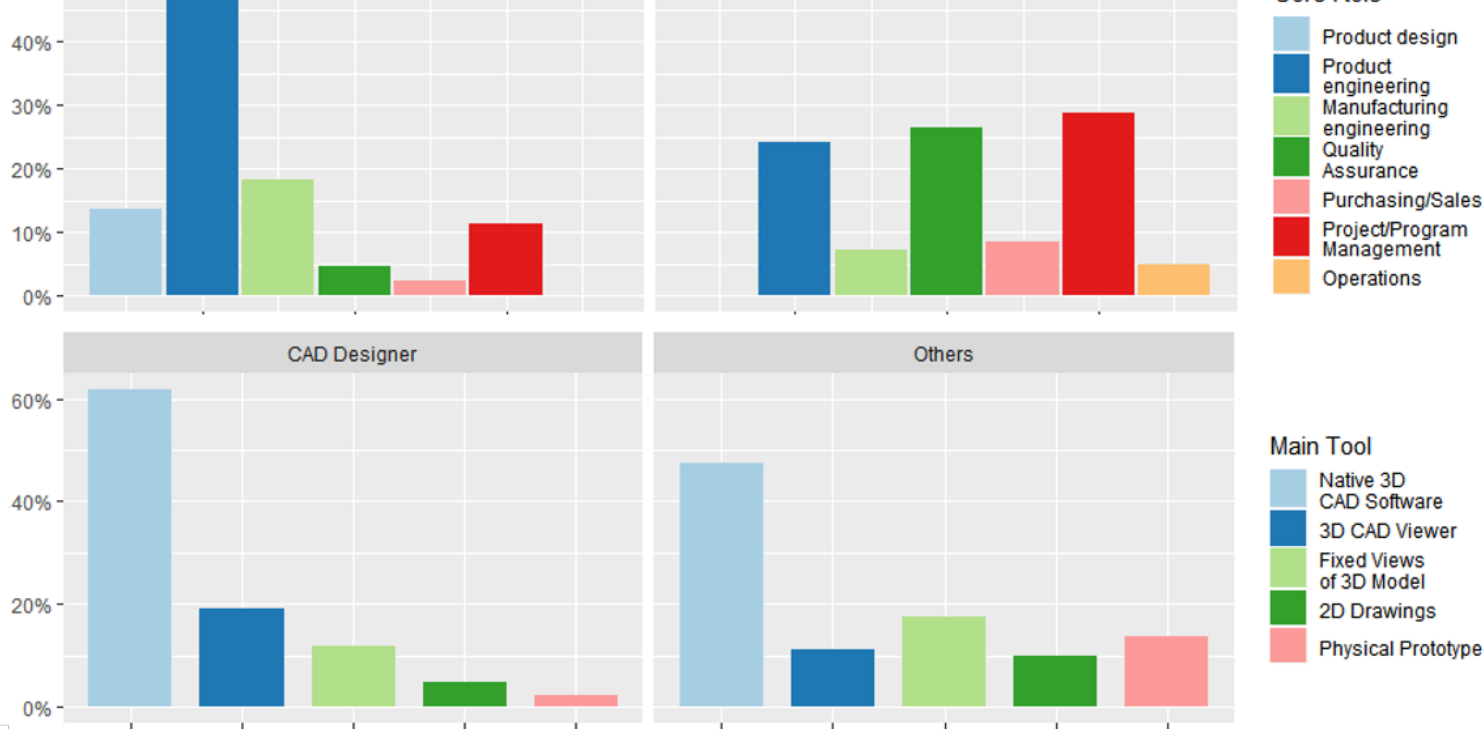

Figure 4. Survey responses tabulated by the respondent's $C A D$ designer status: $C A D$ designer or others. CAD designers self-identified, while others were defined as having no $C A D$ experience at all, or having a basic understanding of CAD and use CAD viewers but do not design in a professional capacity.

Another significant finding is that those that are not CAD Designers are significantly less likely to have access to the company's CAD database. The lack of access to up-to-date CAD models could hinder those that are not CAD Designers' ability to give feedback during design reviews or to voice any concerns they may have about the design at hand. Given the claim of Gul et al., (2013) that individuals' ability to manipulate shared 3D models in real-time from their own viewpoint facilitates coordination of collaborative design, rapid consensus and decision making, access to and fluency with 3D CAD tools is important for design reviewers. Future work could explore how organizations strike the delicate balance between providing too much and not enough information to their employees.

We report a striking predominance of co-located live meetings, accounting for over half of the responses $(52 \%)$. This result is contrary to the original expectation of an increased share of remote meetings, especially considering that multi-national companies account for approximately $70 \%$ of the responses. Furthermore, bearing in mind the industry sectors involved, we may assume that most of these companies often participate in design projects with external parties in the project team. This finding aligns with discussions of the slow uptake of ubiquitous CAD software in general (Horváth and Vroom 2015). While our study investigates CAD Designers' level of access to CAD tools, others have identified other important means of communication used at design reviews, such as a connectivity model (Ariyo et al., 2010). A connectivity model, such as a design structure matrix, provides an architectural view of the product, useful for discussion and scoping. Alternatively, other work proposes centering the design review around a Failure Modes Effects Analysis (Yuichi et al., 2011). It is clear that design researchers believe that the design reviewers would benefit from a common, shared model of the product under review. There is more work to be done to understand which model is appropriate in which context. 
Future work is needed to better understand the differences between CAD Designers and Others in the engineering design context, so that organizations can provide the correct information access conducive to design processes.

The difference in tool preference between CAD Designers and Others is an interesting area to explore further in future work. Answers to how people with different roles best receive information and how different tools can benefit design teams in different phases of the design process can be highly valuable to organizations involved in a wide range of design efforts. Future work could be used to establish guidelines for project managers to better structure and prepare design reviews with the right individuals and right tools. Studies have explored the potential of virtual reality environments in design review activities, an intriguing idea for information sharing and evaluation (Aromaa et al., 2012, Freeman et al., 2016, Casenave and Lugo 2018). Future work could also be used to inform CAD system designers to create tools better suited for stages prior to and beyond the detailed design phase, or create workflows that are more friendly to non-native CAD users.

Recent studies have explored the power of crowdsourced design critiques, in particular exploring whether non-expert reviews are useful (Luther et al., 2015). This study found that aggregated non-expert ratings approach expert critiques and that designers were enthusiastic about crowd-provided critiques and willing to let the feedback influence their design. More should be explored in order to consider the unorthodox crowdsourcing of design reviews at new product development organizations.

Our study also finds that despite the prevalence and availability of virtual collaboration software, the majority of design reviews remain in-person, especially during the earlier phases. Later stages in the design process seem to be more suited for non-live meetings. Future work could be carried out to study how the needs of design teams change throughout the design process and identify the ideal communication media for different phases. Schmidt et al., (2009) found that the number of review decision makers increases over the course of a new product development process. Perhaps this is why we see a trend away from in-person meetings; there is a need to accommodate more stakeholders, less likely to be local. As more stakeholders and parties become involved in the design effort, different communication patterns may emerge that require different tools and communication channels. As companies become increasingly globalized, the ability to effectively leverage the correct tools to coordinate design efforts between multiple sites could help improve efficiency and competitiveness.

Our findings suggest that there are real differences between the characteristics of design reviews at different phases of the product development process. This suggests that studies of design reviews should be context-specific, since design reviews at different phases involve different priorities and settings. This agrees with the findings of Schmidt et al., (2009) who find that the number of decision criteria increases over the course of the new product development process.

\subsection{Limitation of results}

It should be noted that there are limiting factors of this study that need to be taken into consideration before assuming generalizability of the findings. First, as noted in the overview of the dataset, the majority of the respondents are from Brazil. As well, the majority of the responses collected are from the automotive industry.

We completed a robustness check of our results by removing each of these groups from the sample and checking to see if our findings are consistent. In general, we found consistent trends, with a few exceptions: 1) the finding of reduced CAD Access for Others when compared to CAD Designers is not statistically significant in the sample with responses from the automotive industry or Brazil removed there are higher levels of CAD access in the non-automotive industry for DR participants; 2) Outside of the automotive industry, the location of design reviews held during the production phase are proportionally more likely to be co-located live. These differences likely reflect idiosyncrasies in the automotive industry that are not shared with other industries, for example multi-stakeholder production processes. Different geographical regions also could have different approaches to solving problems that may reduce the generalizability of our findings. We encourage future work to replicate and generalize our findings in different industries and geographies.

Our study did not include gender or other demographic information of the survey respondents. Without this information we cannot make comparisons between genders for our survey findings. Important 
future work could be done to confirm the validity and generalizability of our findings and explore any differences that may exist between different genders' responses.

\section{CONCLUSION}

In this paper, we presented the results of a survey of new product development practitioners regarding their design review experiences. We found that the goals and type (location / synchronicity) of design reviews change over the course of a product development project. We found that the majority of design review meetings continue to be held as co-located, live, in-person meetings. For reviewing 3D models, we found that a native 3D CAD package is the most commonly used tool. We found a difference between CAD Designers (more likely to be product engineers) and others, and their access to CAD, as well as their preference for which tool to use at the design review for 3D model evaluation. We hope that our findings spark future work related to better understanding of design reviews and design reviewers in context. Design reviews are an important part of industrial product development processes, so we believe future studies have a large potential to improve these design activities.

\section{REFERENCES}

Adams, R. S., Cardella, M. and Purzer, e. (2016), “Analyzing design review conversations: Connecting design knowing, being and coaching", Design Studies, Vol. 45, pp. 1-8.

Ariyo, O., Heisig, P., Wilson, P., Harnden, M. and Clarkson, P. (2010), "Using Connectivity Models to Support Design Reviews”, DSM 2010 - 12th International DSM Conference, (July), pp. 263-277.

Aromaa, S., Leino, S. P., Viitaniemi, J., Jokinen, L. and Kiviranta, S. (2012), "Benefits of the use of virtual environments in product design review meeting", Proceedings of International Design Conference, DESIGN, Vol. DS 70, pp. 355-364.

Barczak, G. (2012), “The Future of NPD / Innovation Research", The Journal of Product Innovation Management, Vol. 29 No. 3, pp. 355-357.

Carlile, P. R. (2002), “A Pragmatic View of Knowledge and Boundaries: Boundary Objects in New Product Development", Organization Science, Vol. 13 No. 4, pp. 442-455.

Casenave, L. D. and Lugo, J. E. (2018), "Effects of Immersion on Virtual Reality Prototype Design Reviews of Mechanical Assemblies", in Proceedings of the ASME 2018 International Design Engineering Technical Conferences \& Computers and Information in Engineering Conference IDETC/CIE 2018, pp. 1-11.

Edmondson, A. C. and Nembhard, I. M. (2009), "Product Development and Learning in Project Teams: The Challenges Are the Benefits", The Journal of Product Innovation Management, Vol. 26, pp. 123-138.

Freeman, I. J., Salmon, J. and Coburn, J. Q. (2016), "CAD Integration in Virtual Reality Design Reviews for Improved Engineering Model Interaction", in Proceedings of the ASME 2016 International Mechanical Engineering Congress and Exposition IMECE2016, pp. 1-10.

Germani, M., Mengoni, M. and Peruzzini, M. (2012), “An approach to assessing virtual environments for synchronous and remote collaborative design", Advanced Engineering Informatics, Vol. 26 No. 4, pp. 793-813.

Gul, F. L., Wang, X. and Kim, M. J. (2013), “A Framework of collaborative virtual environment for design research", International Journal of Computer Research, Vol. 20 No. 1, pp. 103-121.

Horváth, I. and Vroom, R. W. (2015), "Ubiquitous computer aided design: A broken promise or a Sleeping Beauty?”, CAD Computer Aided Design, Vol. 59, pp. 161-175. http://dx.doi.org/10.1016/j.cad.2014.10.006

Huet, G., Culley, S. J., McMahon, C. A. and Fortin, C. (2007), "Making sense of engineering design review activities", Ai Edam, Vol. 21 No. 3, pp. 243-266.

Krishnan, V. and Ulrich, K. T. (2001), "Product Development Decisions: A Review of the Literature", Management Science, Vol. 47 (February 2015), pp. 1-21.

Luther, K., Tolentino, J.-L., Wu, W., Pavel, A., Bailey, B. P., Agrawala, M., Hartmann, B. and Dow, S. P. (2015), "Structuring, Aggregating, and Evaluating Crowdsourced Design Critique", Proceedings of the 18th ACM Conference on Computer Supported Cooperative Work \& Social Computing - CSCW'15 pp. 473-485.

Ostergaard, K. J., Wetmore III, W. R., Divekar, A., Vitali, H. and Summers, J. D. (2005), “An experimental methodology for investigating communication in collaborative design review meetings", Co-Design, Vol. 1 No. 3, pp. 169-185.

Schmidt, J. B., Sarangee, K. R. and Montoya, M. M. (2009), "Exploring New Product Development Project Review Practices", Journal of Product Innovation Management, Vol. 26, pp. 520-535.

Ulrich, K. T. and Eppinger, S. D. (2015), Product Design and Development, 6 edn, McGraw-Hill, New York, New York, USA. 
Wetmore III, W. R., Summers, J. D. and Greenstein, J. S. (2010), "Experimental study of influence of group familiarity and information sharing on design review effectiveness", Journal of Engineering Design, Vol. 21 No. 1, pp. 111-126.

Yuichi, O., Sho, T., Hirokazu, S. and Yoshiharu, M. (2011), "Empirical consideration of predicting chain failure modes in product structures during design review process", in International Conference on Engineering Design, ICED11, Vol. 4, pp. 278-287.

\section{ACKNOWLEDGMENTS}

The authors would like to thank Vrushank Phadnis and Angie Kim for their insightful feedback on the survey design and analysis. 